\title{
Open-Source Strategy to Enhance Imaginative Intensity and Profits
}

\author{
Nobuyuki Fukawa, Yanzhi Zhang, and Sunil Erevelles
}

\begin{abstract}
The Android project is one of the recent most successful open-source projects. In the Android community, the open-source firm (Google) and application developers co-create value through developing applications for the Android operating system provided by the open-source firm, and share profit from the sales of applications. Alternatively, using a closed-source strategy, a firm could keep the operating system proprietary and sell it to end users. As the first paper to apply the concept of imaginative intensity to analyze the benefits of an open-source strategy, we offer a new explanation for a firm's selection of an open-source strategy over a closed-source strategy. We propose a model to investigate how a for-profit organization utilizes an open-source strategy, in contrast to a closed-source strategy, to enhance imaginative intensity and consequently profit. Our model suggests that an open-source strategy is more effective to manage the diminishing value of ideas than a closed-source strategy.
\end{abstract}

\footnotetext{
N. Fukawa $(\bowtie) \cdot$ Y. Zhang

Missouri University of Science and Technology, Rolla, MO, USA

e-mail: fukawan@mst.edu; zhangyanz@mst.edu

S. Erevelles

University of North Carolina at Charlotte, Charlotte, NC, USA

e-mail: serevell@uncc.edu
} 